\title{
MELD score and antibiotics use are predictors of length of stay in patients hospitalized with hepatic encephalopathy
}

\author{
Valérie Martel-Laferrière ${ }^{1,2^{*}}$, Caitlin Homberger ${ }^{1}$, Kian Bichoupan ${ }^{1}$ and Douglas T Dieterich ${ }^{1}$
}

\begin{abstract}
Background: Hepatic encephalopathy (HE) represents a significant burden to the healthcare system. The aim of this study was to determine factors influencing the hospital length of stay among patients hospitalized with HE.

Methods: A data warehouse query was performed to identify 316 patients with a first hospitalization during which HE occurred, between April 2010 and February 2012. Baseline and hospitalization characteristics were collected with IRB approval. A negative binomial multivariable model was used to control for potential confounders on the length of hospitalization.

Results: Median age was 59 years, and $60.4 \%$ of admitted patients were male. The median MELD score was 22 (IQR: 17-28). Median length of stay was 8 days (IQR: 3.25-14.25). After controlling for MELD score, female gender (2.2 days; $p=0.04$ ), being initially admitted for a reason other than HE (liver-related: 7.6 days; $p<0.01$ and non liver-related 10.7 days; $p<0.01)$ and receiving antibiotics other than rifaximin $(10.5$ days; $p<0.01)$ were associated with longer length of stay whereas hepatitis $C(-3.1$ days; $p<0.01)$ was associated with a shorter length of stay.

Conclusions: MELD score, gender, use of antibiotics other than rifaximin, reason for admission and hepatitis $C$ are predictors readily available in clinic that can help identify patients at risk for longer length of stay.
\end{abstract}

Keywords: Hepatic encephalopathy, Length of stay, MELD score

\section{Background}

Hepatic encephalopathy (HE) is a common complication of chronic liver disease, occurring in $30-45 \%$ of patients with cirrhosis [1,2]. Hepatic encephalopathy is a spectrum of neuropsychiatric manifestations ranging from psychomotor difficulties to altered consciousness and even coma [3].

Each year $\mathrm{HE}$ is responsible for $0.33 \%$ of all hospitalizations, due to the susceptibility of a large population of persons with chronic liver disease, which comprises some 5.5 million people in the United States alone [2,4]. HE is an expensive disease. Among hepatitis $\mathrm{C}(\mathrm{HCV})$ infected patients, HE costs are estimated at $\$ 16,430$ for the first year and $\$ 3,810$ in the subsequent years [5]. For those

\footnotetext{
* Correspondence: valerie.martel-laferriere.chum@ssss.gouv.qc.ca 'Department of Medicine, Division of Liver Diseases, Icahn School of Medicine at Mount Sinai, One Gustave L. Levy Place, New York, NY 10029, USA

${ }^{2}$ Département de microbiologie et maladies infectieuses et Centre de Recherche du Centre Hospitalier de I'Université de Montréal, 1058 St-Denis,
} Montréal H2X 3J4, Qc, Canada requiring hospitalization, length of stay is long, lasting 8.5 days on average, and costs approximately $\$ 63,000$ per case [2]. HE-related hospitalizations are responsible for approximately 7.2 billion dollars in direct costs annually [2]. Fuelled by the aging of the HCV population and the growing incidence of non-alcoholic fatty liver disease, recent trends in the costs of HE-related hospitalization will likely continue.

To better understand the factors underlying the upward trend of hospitalizations and associated costs, this retrospective chart review investigates the length of stay of patients hospitalized with hepatic encephalopathy at a large academic tertiary care center.

\section{Methods \\ This is a retrospective chart-review study conducted at Mount Sinai Medical Center, a large tertiary care center. The Icahn School of Medicine at Mount Sinai Institu- tional Review Board approved the study (GCO \#12-0998).}


A cohort of patients potentially hospitalized with hepatic encephalopathy was collected through a query of the electronic medical record using the ICD-9 codes 570.X, 572.2, 348.3, 348.31, 348.39 and 291.2. The charts of identified patients were reviewed to confirm study eligibility. Patients included were adults hospitalized between April $1^{\text {st }}$ 2010 and February $28^{\text {th }} 2012$ for hepatic encephalopathy or for which hospitalization for another reason was complicated by hepatic encephalopathy. Children with hepatic encephalopathy and adults with metabolic encephalopathy unrelated to liver diseases were excluded. One event per patient was recorded. If more than one event occurred, the first event during the study period was recorded. Data were collected from electronic discharge summaries that included a full history, a list of interventions, lab results, medication profiles and discharge orders.

Patients could present with more than one baseline liver disease. HE hospitalizations were those for which HE was one of the primary diagnoses in the discharge summary. Liver-related hospitalizations were those for which a cirrhosis complication such as ascites or variceal bleeding was the primary diagnosis whereas non liver-related hospitalizations encompassed a large array of reasons of hospital admission such as infection or trauma. HE was not part of the primary diagnosis, but occurred during the hospitalization when the patient was categorized as "liver-related" or "non-liver related". Length of stay was calculated from the day of admission to the day of discharge. If the patient was transferred from another hospital, the days spent in the other hospital were also counted. Thirty-day readmissions were calculated from the day of discharge. Patients who died during the first admission were excluded from the readmission analysis. Model for End-Stage Liver Disease (MELD) score used was the crude MELD score based only on laboratory values (creatinine, International Normalized Ratio (INR) and bilirubin). Choice of treatment was up to the discretion of the clinician as Mount Sinai hospital does not have an HE protocol.

Descriptive statistics were used to investigate the outcomes of hospitalization. Factors influencing the length of hospitalization were identified with a univariable negative binomial regression model. A negative binomial regression was used due to over dispersion. All factors assessed were selected a priori. To control for potential confounders on the length of hospitalization, a multivariable negative binomial regression model was built. All variables with a $\mathrm{p}$-value of $<0.10$ in univariable analysis were assessed in a multivariable model. Variables with a p-value less than 0.05 were retained in the final model. A second negative binomial model that excluded patients who died during the hospitalization, was created. A p-value less than 0.05 was considered statistically significant. Data were analyzed in SAS version 9.3.

\section{Results}

\section{Baseline characteristics}

The medical record query lead to the identification of 502 potential patients, of which 316 were confirmed to fit the inclusion criteria after chart review. The main reasons for exclusion were: age $<18$, or hospitalization for metabolic encephalopathy not related to liver disease.

Table 1 presents the baseline characteristics of the patients. The median age was 59 (IQR: 53-65), more patients were male than female $(191,60.4 \%)$ and a third were Caucasian (107, 33.9\%). MELD scores were high with a median of 22 (IQR: 17-28). Hepatitis $C$ was the most common underlying liver condition (110 patients, $35 \%)$ followed by alcohol (98, 31\%). Only 15 patients (4.7\%) had a transjugular intrahepatic portosystemic shunt (TIPS). For more than half the cohort, the hospitalization recorded was not the first episode of $\mathrm{HE}$ (161/289; 55.7\%).

Hepatic encephalopathy was the main reason for hospital admission for 198 patients (62.7\%). Of the remaining patients, 88 patients (27.8\%) were hospitalized for a liverrelated reason and 30 patients $(9.5 \%)$ for a non liverrelated reason. A small number of patients had acute hepatitis $(32 ; 10.1 \%)$ or spontaneous bacterial peritonitis (34; 10.8\%) during the hospitalization. Most patients received a combination of rifaximin and lactulose (240; 75.9\%).

\section{Hospitalization outcomes}

Sixty-six patients (20.9\%) died during the initial hospitalization. Of the 250 patients discharged from their first hospitalization, 96 (38.4\%) were readmitted within 30 days and an additional 34 patients were readmitted between 31 and 90 days for a total of 130 patients (52\%) readmitted within 90 days. Overall, only $38 \%$ of the patients had a favourable outcome after their initial admission, meaning they did not die in the hospital or were not readmitted within 90-days.

\section{Predictors of length of hospitalization}

The median length of stay was 8 days (IQR: 3.3-14.8 days), but the data contained outliers such as one hospitalization of 113 days. The median length of stay for various subgroups can be seen in Table 2. In univariable analysis, females, patients with acute hepatitis, patients with no prior history of HE or TIPS, and patients who required antibiotics other than rifaximin were more likely to have a longer length of hospitalization (Table 3). The baseline liver disease and the reason for admission also significantly influenced the duration of hospitalization.

In multivariable analysis, we found the change in length of stay to be associated with female gender (2.2 days; $\mathrm{p}=0.04$ ), primary reason of hospitalization other than HE (liver-related: 7.6 days; $\mathrm{p}<0.01$ and non 
Table 1 Baseline and hospitalization characteristics

\begin{tabular}{l} 
Patients characte \\
\hline Age, median (IQR) \\
Gender (male; \%) \\
Race (\%) \\
- Caucasian \\
- African American \\
- Asian \\
- Hispanic \\
- Unknown
\end{tabular}

Baseline liver disease (\%)

- Hepatitis B

- Hepatitis C

- Alcohol

- Non-alcoholic fatty liver disease

- Autoimmune hepatitis

- Primary biliary cirrhosis/Primary sclerosing cholangitis

- Hemochromatosis

- Post-liver complication

- Cryptogenic/unspecified

Presence of TIPS (\%)

Prior history of HE (\%)

MELD, median (IQR)

Primary reason of admission

$\cdot H E$

- Liver related-complication

- Non-liver related

Acute hepatitis (\%)

Treatment

- Lactulose alone

- Lactulose + rifaximin

- Rifaximin alone

- Other ${ }^{\mathrm{a}}$

Use of antibiotics other than rifaximin during the admission (\%)

Length of stay, days, median (IQR)

${ }^{a}$ Metronidazole, neomycin, supportive

liver-related: 10.7 days; $\mathrm{p}<0.01)$ and use of antibiotics other than rifaximin $(10.5$ days; $\mathrm{p}<0.01)$ after controlling for MELD score. Additionally $\mathrm{HCV}$-infected patients had shorter length of stay compared to patients without $\mathrm{HCV}$ (-3.1 days; $\mathrm{p}<0.01$ ) after controlling for MELD score.

When we excluded patients who died during the hospitalization, the median length of stay was 6 days (IQR: 3-13 days). As in the overall cohort, female gender, MELD score, primary reason of hospitalization other than HE and hepatitis $\mathrm{C}$ significantly influenced the length of stay.
Table 2 Length of hospitalization

\begin{tabular}{|c|c|}
\hline Patients characteristics & $\begin{array}{l}\text { Length of hospitalization } \\
\text { in days (IQR) }\end{array}$ \\
\hline \multicolumn{2}{|l|}{ Gender } \\
\hline -Male & $6.0(3.0-13.0)$ \\
\hline -Female & $10.0(4.0-17.0)$ \\
\hline \multicolumn{2}{|l|}{ Age } \\
\hline$\leq 29$ & $9.0(5.0-47.0)$ \\
\hline $30-49$ & $9.5(4.0-16.0)$ \\
\hline $50-69$ & $7.0(3.0-15.0)$ \\
\hline$\geq 70$ & $9.0(3.0-13.0)$ \\
\hline \multicolumn{2}{|l|}{ MELD score } \\
\hline$\leq 10$ & $8.0(3.0-21.0)$ \\
\hline $11-20$ & $4.0(2.0-10.0)$ \\
\hline $21-30$ & $10.0(5.0-16.0)$ \\
\hline $31-40$ & $11.0(6.0-27.0)$ \\
\hline$>40$ & $10.5(5.5-24.5)$ \\
\hline Hepatitis B & $7.5(2.0-11.0)$ \\
\hline Hepatitis C & $5.0(3.0-10.0)$ \\
\hline Alcohol & $9.0(4.0-17.0)$ \\
\hline Non-alcoholic fatty liver disease & $7.0(4.0-15.0)$ \\
\hline Autoimmune hepatitis & $11.5(8.0-17.0)$ \\
\hline PBC/PSC & $7.5(2.0-20.0)$ \\
\hline Hemochromatosis & $10.5(10.0-11.0)$ \\
\hline Post-liver transplant complication & $12.0(5.0-13.0)$ \\
\hline Cryptogenic/unspecified & $10.0(5.0-24.0)$ \\
\hline \multicolumn{2}{|l|}{ Reason of hospitalization } \\
\hline$-H E$ & $5.0(3.0-11.0)$ \\
\hline -Liver-related & $13.0(7.0-22.8)$ \\
\hline -Non liver-related & $13.0(7.8-24.0)$ \\
\hline Acute hepatitis & $14.0(10.3-23.8)$ \\
\hline TIPS & $4.0(1.0-14.0)$ \\
\hline Prior HE & $5.0(3.0-12.0)$ \\
\hline Rifaximin & $8.0(3.0-15.0)$ \\
\hline Use of other antibiotics & $11.0(5.0-21.0)$ \\
\hline
\end{tabular}

In addition, TIPS $(-4.5$ days; $\mathrm{p}<0.04)$ and primary biliary cirrhosis/primary sclerosing cholangitis $(-8.3$ days; $\mathrm{p}<$ 0.01 ) were associated with a shortened length of stay.

\section{Discussion}

Overall, clinical outcomes following a hospitalization for hepatic encephalopathy in a tertiary care liver transplant center were discouraging. Hospitalizations were long with a median of 8 days but a maximum up to 113 days and 33 patients (10.4\%) hospitalized for longer than one month. In-patient mortality and 30-day readmissions were also frequent $(20.9 \%$ and $38.6 \%$, respectively). These 
Table 3 Predictors of length of hospitalization

\section{A. Overall cohort}

\begin{tabular}{|c|c|c|c|c|}
\hline & \multicolumn{2}{|l|}{ Univariable analysis } & \multicolumn{2}{|l|}{ Multivariable analysis } \\
\hline & Change in length of stay (days) & p-value & Change in length of stay (days) & p-value \\
\hline Gender (female) & 5.1 & $<0.01$ & 2.2 & 0.04 \\
\hline \multicolumn{5}{|l|}{ Age } \\
\hline$\leq 29$ & Reference & . & & \\
\hline $30-49$ & -10.2 & 0.19 & & \\
\hline $50-69$ & -12.5 & 0.06 & & \\
\hline$\geq 70$ & -13.8 & 0.04 & & \\
\hline \multicolumn{5}{|l|}{ MELD score } \\
\hline$\leq 10$ & Reference & . & Reference & . \\
\hline $11-20$ & -6.9 & 0.01 & -5.4 & $<0.01$ \\
\hline $21-30$ & -1.8 & 0.59 & -3.8 & 0.02 \\
\hline $31-40$ & 3.1 & 0.48 & -2.9 & 0.15 \\
\hline$>40$ & 4.9 & 0.38 & -2.5 & 0.33 \\
\hline Hepatitis B & -0.2 & 0.95 & . & . \\
\hline Hepatitis C & -6.1 & $<0.01$ & -3.1 & $<0.01$ \\
\hline Alcohol & 1.0 & 0.53 & ${ }^{\circ}$ & . \\
\hline Non-alcoholic fatty liver disease & 1.3 & 0.60 & . & \\
\hline Autoimmune hepatitis & -1.4 & 0.73 & . & . \\
\hline $\mathrm{PBC} / \mathrm{PSC}$ & 3.1 & 0.50 & . & . \\
\hline Hemochromatosis & -2.1 & 0.67 & . & . \\
\hline Post-liver transplant complication & 3.2 & 0.51 & & \\
\hline Cryptogenic/unspecified & 6.1 & $<0.01$ & . & . \\
\hline \multicolumn{5}{|l|}{ Reason of hospitalization } \\
\hline$-H E$ & Reference & . & Reference & . \\
\hline -Liver-related & 9.8 & $<0.01$ & 7.6 & $<0.01$ \\
\hline -Non liver-related & 12.9 & $<0.01$ & 10.7 & $<0.01$ \\
\hline Acute hepatitis & 9.7 & $<0.01$ & . & . \\
\hline TIPS & -3.5 & $<0.01$ & . & \\
\hline Prior HE & -3.7 & 0.01 & . & . \\
\hline Rifaximin & 1.9 & 0.28 & . & \\
\hline Use of other antibiotics & 10.2 & $<0.01$ & 10.5 & $<0.01$ \\
\hline \multicolumn{5}{|l|}{ B. Survivors cohort } \\
\hline & \multicolumn{2}{|l|}{ Univariable analysis } & \multicolumn{2}{|l|}{ Multivariable analysis } \\
\hline & Change in length of stay (days) & p-value & Change in length of stay (days) & p-value \\
\hline Gender (female) & 5.5 & $<0.01$ & 3.4 & 0.01 \\
\hline \multicolumn{5}{|l|}{ Age } \\
\hline$\leq 29$ & Reference & Ref & . & \\
\hline $30-49$ & 1.5 & $<0.01$ & & \\
\hline $50-69$ & 3.2 & $<0.01$ & & \\
\hline$\geq 70$ & 5.1 & & & \\
\hline \multicolumn{5}{|l|}{ MELD score } \\
\hline$\leq 10$ & Reference & Ref & Reference & Reference \\
\hline $11-20$ & -8.0 & $<0.01$ & -6.6 & $<0.01$ \\
\hline
\end{tabular}


Table 3 Predictors of length of hospitalization (Continued)

\begin{tabular}{|c|c|c|c|c|}
\hline $21-30$ & -2.7 & 0.41 & -5.1 & $<0.01$ \\
\hline $31-40$ & 5.2 & 0.33 & 2.7 & 0.38 \\
\hline$>40$ & 15.6 & 0.13 & 3.0 & 0.52 \\
\hline Hepatitis B & -4.1 & $<0.01$ & . & . \\
\hline Hepatitis C & -6.1 & $<0.01$ & -3.9 & $<0.01$ \\
\hline Alcohol & 3.2 & 0.04 & . & . \\
\hline Non-alcoholic fatty liver disease & 2.7 & 0.30 & . & . \\
\hline Autoimmune hepatitis & -1.4 & 0.74 & . & . \\
\hline $\mathrm{PBC} / \mathrm{PSC}$ & -8.2 & 0.01 & -8.3 & $<0.01$ \\
\hline Hemochromatosis & -0.8 & 0.86 & . & . \\
\hline Post-liver transplant complication & 1.9 & 0.72 & & \\
\hline Cryptogenic/unspecified & 6.0 & 0.01 & . & . \\
\hline \multicolumn{5}{|l|}{ Reason of hospitalization } \\
\hline$-H E$ & Reference & Ref & Reference & Ref \\
\hline -Liver-related & 21.8 & $<0.01$ & 10.3 & $<0.01$ \\
\hline -Non liver-related & 27.7 & $<0.01$ & 15.9 & $<0.01$ \\
\hline Acute hepatitis & 16.0 & $<0.01$ & & \\
\hline TIPS & -6.8 & $<0.01$ & -4.5 & 0.04 \\
\hline Prior HE & -2.9 & 0.04 & . & . \\
\hline Rifaximin & -0.45 & 0.81 & . & . \\
\hline Use of other antibiotics & 9.4 & $<0.01$ & 9.9 & $<0.01$ \\
\hline
\end{tabular}

numbers are slightly higher than previously reported mortality rates of $14.1-15.6 \%$ and 30 -day readmission rates of $20 \%[2,6]$.

We identified potential predictors of longer length of stay in our patient population. As expected, the length of stay in patients was negatively associated with MELD. Overall, patients with lower MELD had shorter length of stay, except for patients with MELD $<10$. HE is rare in patients with low MELD and hospitalization length may have been driven by another cause than HE.

To our knowledge, no other studies on the association between length of stay and infections or antibiotic use have been published to date. Although antibiotic use is likely a marker of sicker patients, it remains significant even after adjustment for MELD score. The impact of antibiotics on length of stay could originate from two pathways: a modification of the gut flora induced by the antibiotics or as a surrogate marker for infections. The latter is more probable as infection is common in patients with advanced liver disease and is a frequent trigger of HE [7].

In the past, infections have been associated with poor outcomes in patients with HE. Hung et al. retrospectively looked at 4,150 patients with HE and cirrhosis [8]. In a Cox proportional-hazards regression model, they found a significant association between the presence of a coexisting infection and 30-day, 30-90-day and 90-day-1 year mortality (HR: 1.66 (95\% CI: 1.32-1.94), 1.51 (95\% CI
1.23-1.85), 1.34 (95\% CI 1.13-1.58), respectively) while adjusting for age, gender, $\mathrm{HCV}$, variceal bleeding, ascites, alcoholism, acute and chronic renal failure and peptic ulcer bleeding [8]. In a recent prospective double-blind randomized controlled trial comparing lactulose with placebo to lactulose with rifaximin, higher baseline leukocyte count was associated with non-response to treatment [9]. Similarly, Sharma et al. found higher leukocyte counts among non-lactulose responders than among responders, but they did not find an association between infection and non-response except for the occurrence of spontaneous bacterial peritonitis and related leukocytes elevation to systemic inflammation of patients with advanced liver disease [10]. Shawcross et al. did not find any difference in survival between patients with and without positive cultures when they looked at 100 patients admitted to the intensive care unit for HE [11].

Admission for HE was associated with a shorter length of stay. It could be hypothesized that HE, as well as possible precipitants, were rapidly identified and treated in these patients. Similarly, among survivors, patients with TIPS had shorter length of stay. As HE is a frequent complication of TIPS (median cumulative 1-year incidence: $10-50 \%)$, it was likely sought after and managed more aggressively in these patients [12].

Patients with HCV liver disease had a shorter length of stay than patients with non-HCV liver disease. The reason 
for this observation is unclear. One explanation could be a slower and often more predictable progression of $\mathrm{HCV}$-induced liver disease compared to other liver conditions. This is also supported by the fact that patients with primary biliary cirrhosis/primary sclerosing cholangitis, also slowly progressing diseases, had shorter lengths of stay in the survivors multivariable analysis. Wiegand et al. also evoked a difference in disease progression to explain clinical differences observed in type of decompensation between patients with alcoholic vs. non-alcoholic liver diseases [13].

\section{Conclusions}

Female gender, hepatitis $\mathrm{C}$ infection, reason of admission, MELD score and use of antibiotics are predictors of length of stay. Because these characteristics are available to the clinicians rapidly after patient admission, they can be used to target interventions for patients at high risk and reduce both length of stay and readmission. Even if limited by their retrospective nature, our data are derived from a large cohort of patients and include patients with advanced liver disease (median MELD of 22).

\section{Abbreviations}

HCV: Hepatitis C; HE: Hepatic encephalopathy; TIPS: Transjugular intrahepatic portosystemic shunt; MELD: Model for End-stage Liver Disease;

INR: International Normalized Ratio; PBC: Primary biliary cirrhosis; PSC: Primary sclerosing cholangitis; IQR: Interquartile range.

\section{Competing interests}

Valérie Martel-Laferrière: research grant: Salix Pharmaceuticals, Caitlin Homberger: nothing to disclose, Kian Bichoupan: nothing to disclose, Douglas T. Dieterich: research grant: Salix Pharmaceuticals.

\section{Author contributions}

VML: study concept and design; acquisition of data; analysis and interpretation of data; drafting of the manuscript; statistical analysis; study supervision. CH: study concept and design; acquisition of data; analysis and interpretation of data; drafting of the manuscript. KB: study concept and design; analysis and interpretation of data; drafting of the manuscript; critical revision of the manuscript for important intellectual content; statistical analysis. DTD: study concept and design; interpretation of data; critical revision of the manuscript for important intellectual content; study supervision. All authors read and approved the final manuscript.

\section{Acknowledgements}

No funding was directly received for this work.

Valérie Martel-Laferrière was funded by the 2012 Grant of the CHUM

Foundation.

Statistical support was provided by Salix Pharmaceutical in an initial version of this work, but not in its current format.

Received: 24 June 2014 Accepted: 9 October 2014

Published: 17 October 2014

\section{References}

1. Poordad FF: Review article: the burden of hepatic encephalopathy. Aliment Pharmacol Ther 2007, 25(Suppl 1):3-9. doi:10.1111/j.1746-6342.2006.03215.x [published Online First: Epub Date]

2. Stepanova M, Mishra A, Venkatesan C, Younossi ZM: In-hospital mortality and economic burden associated with hepatic encephalopathy in the United States from 2005 to 2009. Clin Gastroenterol Hepatol 2012, 10(9):1034-1041. el doi:10.1016/j.cgh.2012.05.016 [published Online First: Epub Date]
3. Ferenci P, Lockwood A, Mullen K, Tarter R, Weissenborn K, Blei AT: Hepatic encephalopathy-definition, nomenclature, diagnosis, and quantification: final report of the working party at the 11 th World Congresses of Gastroenterology, Vienna, 1998. Hepatology 2002, 35(3):716-721. doi:10.1053/jhep.2002.31250 [published Online First: Epub Date]

4. Kim WR, Brown RS Jr, Terrault NA, El-Serag H: Burden of liver disease in the United States: summary of a workshop. Hepatology 2002, 36(1):227-242. doi: 10.1053/jhep.2002.34734 [published Online First: Epub Date]

5. El Khoury AC, Klimack WK, Wallace C, Razavi H: Economic burden of hepatitis C-associated diseases in the United States. J Viral Hepat 2012, 19(3):153-160. doi:10.1111/j.1365-2893.2011.01563.x [published Online First: Epub Date]

6. Berman K, Tandra S, Forssell K, Vuppalanchi R, Burton JR Jr, Nguyen J, Mullis D, Kwo P, Chalasani N: Incidence and predictors of 30-day readmission among patients hospitalized for advanced liver disease. Clin Gastroenterol Hepatol 2011, 9(3):254-259. doi:10.1016/j.cgh.2010.10.035 [published Online First: Epub Date]

7. Jalan R, Fernandez J, Wiest R, Jalan R, Fernandez J, Wiest R, Schnabl B, Moreau R, Angeli P, Stadlbauer V, Gustot T, Bernardi M, Canton R, Albillos A, Lammert F, Wilmer A, Mookerjee R, Vila J, Garcia-Martinez R, Wendon J, Such J, Cordoba J, Sanyal A, Garcia-Tsao G, Arroyo V, Burroughs A, Gines P: Bacterial infections in cirrhosis: a position statement based on the EASL special conference 2013. J Hepatol 2014, 60(6):1310-1324. doi:10.1016/j.jhep.2014.01.024 [published Online First: Epub Date]|.

8. Hung TH, Lay CJ, Chang CM, Tsai JJ, Tsai CC, Tsai CC: The effect of infections on the mortality of cirrhotic patients with hepatic encephalopathy. Epidemiol Infect 2013, 1-8. doi:10.1017/S0950268813000186 [published Online First: Epub Date]|.

9. Sharma BC, Sharma P, Lunia MK, Srivastava S, Goyal R, Sarin SK: A randomized, double-blind, controlled trial comparing rifaximin plus lactulose with lactulose alone in treatment of overt hepatic encephalopathy. Am J Gastroentero/ 2013, 108(9):1458-1463. doi:10.1038/ajg.2013.219 [published Online First: Epub Date].

10. Sharma P, Sharma BC, Sarin SK: Predictors of nonresponse to lactulose in patients with cirrhosis and hepatic encephalopathy. Eur J Gastroenterol Hepatol 2010, 22(5):526-531. doi:10.1097/MEG.0b013e3283341b7d [published Online First: Epub Date].

11. Shawcross DL, Sharifi Y, Canavan JB, Shawcross DL, Sharifi Y, Canavan JB, Yeoman AD, Abeles RD, Taylor NJ, Auzinger G, Bernal W, Wendon JA: Infection and systemic inflammation, not ammonia, are associated with Grade 3/4 hepatic encephalopathy, but not mortality in cirrhosis. J Hepatol 2011, 54(4):640-649. doi:10.1016/j.jhep.2010.07.045 [published Online First: Epub Date]

12. Vilstrup H, Amodio P, Bajaj J, Vilstrup H, Amodio P, Bajaj J, Cordoba J, Ferenci P, Mullen KD, Weissenborn K, Wong P: Hepatic encephalopathy in chronic liver disease: 2014 Practice Guideline by the American Association for the Study of Liver Diseases and the European Association for the Study of the Liver. Hepatology 2014, 60(2):715-735. doi:10.1002/hep.27210 [published Online First: Epub Date].

13. Wiegand J, Kuhne M, Pradat P, Mossner J, Trepo C, Tillmann HL: Different patterns of decompensation in patients with alcoholic vs non-alcoholic liver cirrhosis. Aliment Pharmacol Therapeut 2012, 35(12):1443-1450. doi:10.1111/j.1365-2036.2012.05108.x [published Online First: Epub Date]

\section{doi:10.1186/1471-230X-14-185}

Cite this article as: Martel-Laferrière et al:: MELD score and antibiotics use are predictors of length of stay in patients hospitalized with hepatic encephalopathy. BMC Gastroenterology 2014 14:185. 